\title{
MODELO DE EVALUACIÓN DEL DESEMPEÑO DOCENTE EN EL AMBITO INSTRUCTIVO DESDE UNA PERSPECTIVA ESTUDIANTIL - CASO: UNIVERSIDAD NACIONAL AGRARIA LA MOLINA
}

\author{
MODEL TO ASSEST TEACHERS IN THE INSTRUCTIVE FIELD FROM STUDENT \\ PERSPECTIVES - CASE: NATIONAL AGRICULTURE UNIVERSITY AT LA MOLINA
}

${ }^{1}$ Fernando Rene Rosas Villena

\begin{abstract}
Resumen
En este artículo se identifica un modelo de dimensiones y variables para la evaluación del desempeño docente en el ámbito instructivo en la Universidad Nacional Agraria La Molina (UNALM). La validez del modelo teórico, así como la validez de constructo de la encuesta estudiantil se determinan con el Análisis Factorial (AF). La confiabilidad de la encuesta estudiantil se determina con el Coeficiente Alfa de Cronbach. La investigación se realizó entre los años 20082014, aplicándose durante este período trece prototipos de encuesta estudiantil. El último modelo de siete factores alcanzó un porcentaje acumulado de variancia de $71.38 \%$ y la encuesta estudiantil un valor de confiabilidad alfa de Cronbach de 0.9336 .
\end{abstract}

Palabras clave: Análisis Factorial, Desempeño Docente.

\begin{abstract}
This article identifies a model with dimensions and variables for the evaluation of the teaching performance in the instructional field at the National Agriculture University at La Molina (UNALM). The validity of the theoretical model, as well as the construct validity of the student survey are determined with the confirmatory Factorial analyses (AFC). The reliability of the student survey is determined by the coefficient alpha of Cronbach. The research was conducted between the years 2008-2014, thirteen student survey prototypes to apply during this period. The latest model of seven factors reached a cumulative percentage of variance of $71.38 \%$ and the student survey a value for of 0.9336 Cronbach Alpha reliability.
\end{abstract}

Keys words: Factor Analysis, Teacher Performance

\section{Introducción}

La evaluación del desempeño docente en las universidades es un recurso utilizado para orientar, corregir, afianzar y consolidar sus actividades. Su objetivo radica en la posibilidad de desarrollar estrategias de capacitación y perfeccionamiento docente que permitan mejorar su calidad académica.

La evaluación docente por parte de los estudiantes es un tema de permanente actualidad, ampliamente debatido y complejo, que ha venido investigándose con cierta intensidad desde hace más de treinta años.

Acevedo (2003) señala la importancia de la evaluación docente en base a dos considerandos: El primero, afirmando que el pensamiento del profesor universitario ejerce influencia en el rendimiento de sus estudiantes. Los profesores son responsables, entre otras cosas, de introducir cambios y matices que modulan el clima del aula, la calidad del trabajo y la orientación de los aprendizajes de los alumnos. En sus manos, actitudes y aptitudes descansa gran parte del éxito de las políticas educativas, estrategias, planes y otros elementos más. El segundo, al señalar que las nuevas tecnologías y los constantes cambios ejercen presión para mejorar su docencia.

Gilmore (1984) señala tres razones que justifican la evaluación del desempeño docente por parte de los alumnos: (1) permite que el alumnado opine sobre sus docentes, originando beneficios políticos para la institución, (2) se considera que los estudiantes, por su naturaleza, son los observadores más directos de la docencia, por lo que están en una posición única para valorar la calidad del curso, la dedicación y la preparación del profesorado y (3) la fiabilidad de las observaciones realizadas por el alumnado es normalmente elevada y depende, entre otros factores, del número de encuestados. En la UNALM las actividades docentes se evalúan en base 
a cinco componentes: (1) enseñanza, (2) investigación, (3) extensión y proyección social, (4) capacitación y (5) labores administrativas. La enseñanza se evalúa a través del desempeño docente en el ámbito instructivo durante el ciclo académico mediante una encuesta estudiantil.

Los profesores son evaluados en tres momentos diferentes: (1) durante su ingreso a la docencia, (2) en cada ciclo académico y (3) en los procesos de ratificación y/o ascenso. La evaluación durante el ingreso a la docencia y en las ratificaciones y/o ascensos se realiza con indicadores y tablas que califican las cinco actividades señaladas. En cambio la evaluación del desempeño docente en un ciclo académico, se circunscribe a un informe elaborado por el propio docente donde se detalla lo realizado en estas cinco actividades. Estos tres tipos de evaluaciones forman parte de la trayectoria docente y en la actualidad no están articuladas dentro de un Sistema General de Evaluación Docente.

La presente investigación tiene como objetivos, validar un modelo de evaluación del desempeño del docente en el ámbito instructivo y elaborar una encuesta estudiantil que permita su medición. Los resultados del estudio contribuirán a sentar las bases para el desarrollo de un Sistema General de Evaluación Docente en la UNALM.

\section{Materiales y métodos}

\section{Tipo de Investigación}

Es confirmatorio y correlacional. Confirmatorio porque la técnica del Análisis Factorial Confirmatorio (AFC) permite validar la participación de los factores que forman parte del modelo propuesto. Correlacional porque el AFC permite verificar si las preguntas que se diseñaron para medirlos se correlacionan en forma efectiva con los factores.

\section{Diseño de la investigación}

Es no experimental de corte transversal. No experimental debido a que no hubo manipulación intencional de una o más variables independientes con el fin de probar su causalidad con una variable dependiente. Transversal porque las encuestas estudiantiles (13) se aplicaron en momentos específicos de tiempo.

\section{Formulación de la hipótesis}

El modelo propuesto de evaluación del desempeño docente en el ámbito instructivo para un ciclo académico desde una perspectiva estudiantil, constituido por loa factores: (1) planificación del curso, (2) dominio del curso, (3) métodos y recursos de instrucción, (4) obligaciones docentes, (5) método evaluativo, (6) motivación e interacción con los alumnos y (7) valoración general, presenta mayor validez que el modelo actual de la UNALM conformado por los factores:(1) Conducción del aprendizaje, (2) Disposición y cumplimiento, (3) Motivación, (4) Evaluación y (5)

\section{Uso de material educativo}

\section{Población y muestra}

Las poblaciones son todos los alumnos de la UNALM matriculados en los ciclos académicos del 2008-I al 2014-I. Se garantizó el tamaño óptimo de las muestras (349) con un nivel de confianza del 90\% y un error de estimación de $\pm 5 \%$.

\section{Metodología aplicada}

El procedimiento habitual para validar un modelo de esta naturaleza es el siguiente: proponer un modelo con un número determinado de factores, elaborar un instrumento de evaluación (encuesta estudiantil) con preguntas apropiadas para medir cada uno de los factores, aplicar el instrumento a los estudiantes, aplicar el AFC y concluir en términos de la validez (autovalor y porcentaje de variancia acumulada) y confiabilidad (Coeficiente Alfa de Cronbach).

Este procedimiento puede dar lugar a conclusiones no satisfactorias cuando se tiene: bajo porcentaje de variancia acumulada, preguntas estadísticamente correlacionadas con el factor pero sin relación lógica de pertenencia en ellos, bajo número de preguntas por factor o cualquier otra combinación de estos resultados.

Por esta razón, el objetivo de la investigación es encontrar un modelo con el menor número de cuestionamientos posibles. La hipótesis de trabajo busca verificar que el nuevo modelo sea mejor que el vigente en términos de validez y confiabilidad.

Se utilizó la técnica del Análisis Factorial (AF) propuesta por Hair, Anderson, Tatham y Black (1999). Debido a que los factores se predeterminaron el AF se usó con fines confirmatorios la técnica pasó a denominarse Análisis Factorial Confirmatorio (AFC).

\section{Primer paso: Objetivos del análisis factorial}

El AF tiene dos propósitos: resumir y reducir información. El propósito de resumir información es condensar la información de las variables originales en otra más pequeña denominada factores. El propósito de reducir información es para utilizar las variables suplentes de una serie de variables más grande en análisis multivariantes posteriores.

\section{Segundo paso: Diseño de un análisis factorial}

En este paso se da respuesta a las siguientes interrogantes: ¿qué tipo de matriz de correlación se debe utilizar?, ¿qué variables se deben considerar y cómo se deben medir?, y ¿cuál es el tamaño de muestra óptimo?. En la primera pregunta se adopta el siguiente criterio: si la agrupación es de variables se utiliza la matriz de correlación " $R$ " y si es de individuos la "Q". En la segunda se debe alcanzar 
un ratio no menor de 10 a 1 y las variables deben ser métricas. En la última se considera por lo menos un número de observaciones cinco veces mayor que el número de variables a ser analizadas.

\section{Tercer Paso: Supuestos del análisis factorial}

Los supuestos de normalidad, homocedasticidad y linealidad son deseables desde el punto de vista teórico, sin embargo, en la práctica se exige verificar el supuesto de multicolinealidad para continuar con el AF. Para ello, se utilizan diferentes indicadores estadísticos como: matriz de $\mathrm{R}$ de correlaciones, matriz de correlación antiimagen, contraste de esfericidad de Bartlett, medidas de suficiencia de muestreo (MSA) y Kaiser Meyer Oikin (KMO). Es aconsejable que un número importante de correlaciones de la matriz $\mathrm{R}$ sean significativas, que los valores de correlación en la diagonal de la matriz antiimagen sean altos, que MSA $>0.5$, que P-value de Bartlett $<0.5$ y $\mathrm{KMO}>0.5$.

Cuarto Paso: La estimación de los factores

En este paso se elige el método de extracción que se va a utilizar (común o componentes principales) y se fija el número de factores que representará la estructura subyacente de información. El método de componentes principales se utiliza si el objetivo es resumir la información y el método común si el objetivo es identificar los factores que representarán la estructura subyacente de información. Existen varios criterios que ayudan a determinar el número de factores a extraer: raíz latente, a priori, porcentaje de la variancia y contraste de caída. Con el criterio de la raíz latente se eligen tantos factores como raíces latentes o autovalores sean mayores a 1, con el criterio a priori el número de factores a extraer están prefijados, con el criterio de porcentaje de la variancia acumulada se considera un número de factores con mayor porcentaje acumulado de variancia, y con el criterio de contraste de caída el número de factores a extraer se determina gráficamente cuando la curva de raíces latentes presenta una rectificación importante en su decrecimiento.

\section{Quinto Paso: Interpretación de los factores}

Se calcula primero la matriz no rotada y sus valores sirven para tener una idea preliminar del número de factores a extraer para su interpretación. Luego, se realiza la rotación de factores respecto a los ejes de referencia (ortogonal $\mathrm{u}$ oblicua), con el fin de lograr soluciones factoriales más simples y teóricamente más significativas.

\section{Resultados y discusión}

La estructura de la investigación se divide en tres etapas. En la primera, se valida el modelo vigente en la UNALM mediante la aplicación del AFC a la encuesta estudiantil del ciclo académico 2008-I (iteración 1). En la segunda, se revisan investigaciones similares con el fin de identificar factores comunes y utilizarlos de base formular nuevos modelos. En la tercera, se realiza el proceso de búsqueda del mejor modelo explicativo durante los ciclos académicos 2008-I al 2014-I (iteración 2 a la 13).

\section{Primera Etapa:}

Se probó la validez del modelo vigente en la UNALM mediante el AFC. Este modelo está conformado por cinco factores: (1) Conducción del aprendizaje, (2) Disposición y cumplimiento, (3) Motivación, (4) Evaluación y (5) Uso de material educativo.

Los resultados de la Tabla 1: Matriz de componentes rotados y Tabla 2: Variancia total explicada, corresponden a la aplicación del AFC a la encuesta estudiantil (15 preguntas) del ciclo académico 2008-I.

Tabla 1. Varianza Total Explicada.

\begin{tabular}{cccc}
\hline Componente & Valores & $\begin{array}{c}\text { Variancia } \\
(\%)\end{array}$ & $\begin{array}{c}\text { Variancia } \\
\text { Acumulada (\%) }\end{array}$ \\
\hline 1 & 10,777 & 24,829 & 24,829 \\
2 & 0,749 & 20,979 & 45,808 \\
3 & 0,537 & 18,465 & 64,273 \\
4 & 0,378 & 13,486 & 77,760 \\
5 & 0,233 & 8,614 & 86,374 \\
\hline
\end{tabular}

Fuente: Elaboración propia

Tabla 2. Matriz de componentes rotados.

\begin{tabular}{cccccc}
\hline Variables & 1 & 2 & 3 & 4 & 5 \\
\hline 1 & 0.302 & 0.369 & 0.789 & 0.173 & 0.126 \\
2 & 0.303 & 0.239 & 0.683 & 0.321 & 0.393 \\
3 & 0.521 & 0.202 & 0.595 & 0.310 & 0.265 \\
4 & 0.320 & 0.292 & 0.395 & 0.257 & 0.707 \\
5 & 0.648 & 0.284 & 0.537 & 0.241 & 0.184 \\
6 & 0.651 & 0.419 & 0.419 & 0.254 & 0.100 \\
7 & 0.728 & 0.345 & 0.271 & 0.216 & 0.252 \\
8 & 0.723 & 0.277 & 0.342 & 0.301 & 0.200 \\
9 & 0.608 & 0.472 & 0.255 & 0.262 & 0.278 \\
10 & 0.493 & 0.521 & 0.089 & 0.380 & 0.403 \\
11 & 0.242 & 0.789 & 0.251 & 0.231 & 0.263 \\
12 & 0.486 & 0.713 & 0.242 & 0.238 & 0.150 \\
13 & 0.292 & 0.737 & 0.392 & 0.287 & 0.078 \\
14 & 0.239 & 0.359 & 0.267 & 0.780 & 0.228 \\
15 & 0.470 & 0.285 & 0.309 & 0.675 & 0.146 \\
\hline
\end{tabular}

Fuente: Elaboración propia

Se puede observar en la Tabla 1 que el Componente 1 registra un autovalor de 10.78 (por encima del óptimo de 1) y un valor de porcentaje de variancia acumulada de $24.83 \%$.

En la Tabla 2 el Componente 1 está integrada por dos factores: Factor 1: Conducción del aprendizaje y Factor 5: Uso de material educativo. Dentro de ella se ubican 2 de las 3 variables diseñadas para medir el Factor 1 y las 2 variables diseñadas para medir el Factor 5. El 
Componente 2, aun cuando su autovalor está por debajo del óptimo (0.75) podría ser considerada como parte del modelo y contribuiría a elevar el porcentaje de variancia acumulada a $45.81 \%$. Los otros tres componentes (3, 4 y 5) presentan autovalores muy alejado del óptimo (menores a 0.6) y no deberían formar parte del modelo.

Como resultado de este análisis se puede afirmar que el modelo de evaluación docente vigente en la UNALM, así como la encuesta estudiantil carece de validez.

Por otro lado, la confiabilidad de la encuesta estudiantil registró un valor Alfa de Cronbach de 0.971 por lo que puede catalogarse como confiable (cercano a 1).

\section{Segunda Etapa:}

Se revisaron investigaciones realizadas en universidades del extranjero sobre este tema con el fin de encontrar los factores con igual o similar denominación. Se identificaron siete factores: (1) planificación del curso, (2) dominio del curso, (3) métodos y recursos de instrucción, (4) obligaciones docentes, (5) método evaluativo, (6) motivación e interacción con los alumnos y (7) apreciación general del docente.

\section{Factor 1: Organización del curso}

Este factor evalúa la percepción estudiantil sobre planificación y organización del curso. Se manifiesta por la estructura lógica de los temas en el programa del curso, el nivel de desarrollo asignado a cada uno de ellos, entre otros aspectos. Este tema ha sido destacado en estudios realizados por Trent y Cohen (1973), Monk (1994), García Ramos (1996, 1997 y 1998) y Acevedo (2003).

\section{Factor 2: Dominio del curso}

Se evalúa la percepción estudiantil sobre el dominio que el docente tiene sobre el curso. Se manifiesta a través del tratamiento claro, ordenado y profundo en el desarrollo de cada una de sus clases y por la soltura y satisfacción que demuestra el profesor en su exposición. Este factor es identificado por Feldman (1984, 1986), Mortimore (1988), Bookover y Lezotte (1997), Gibbs (1992), Centra (1993), Abrami (1997), Marh (2001) y Acevedo (2003).

\section{Factor 3: Métodos y recursos de instrucción}

Este factor recoge la opinión estudiantil sobre el uso adecuado de los métodos y recursos de instrucción. Los métodos de enseñanza se diseñan exclusivamente para este fin (programas específicos, demostraciones, trabajos grupales, etc.) en cambio los recursos son los que diseñados para otro fin son utilizados indirectamente para ayudar en la labor de enseñanza (programas genéricos, multimedia, textos, etc.). Entre las investigaciones que identifican a este factor se tiene: Acevedo (2003), Molero y Ruiz (2005), y Muñoz, Ríos, y Abalde (2013).

\section{Factor 4: Obligaciones docentes}

Valora la opinión estudiantil sobre el cumplimiento de las obligaciones docentes en el ámbito instructivo. Evalúa si el profesor inicia o termina su clase a la hora indicada, si justifica y recupera con prontitud su inasistencia a clase, si cumple con atender las consultas extra clase, entre otros. Este factor es identificado por Molero y Ruiz (2005), Colina, Medina, Parra, Cedróz y Montoya (2008) y Muñoz, Ríos, y Abalde (2013)

\section{Factor 5: Método evaluativo}

Evalúa los siguientes aspectos: elaboración de evaluaciones en correspondencia con el nivel de la clase, objetividad e imparcialidad para evaluar y calificar, periodicidad de las evaluaciones, oportunidad de retroalimentación del aprendizaje a través de la explicación de la calificación y disposición para revisar la calificación. Entre los investigadores que han identificado esta dimensión de la evaluación docente se encuentran: Marsh y Bailey (1993), Lowman (1994) y D’Apollonia y Abrami (1997).

\section{Factor 6: Motivación e interacción con los alumnos}

La motivación e interacción del profesor con sus alumnos son dimensiones de evaluación del desempeño docente que en algunas investigaciones son identificadas como factores independientes y en otras como un solo factor. La "motivación" es identificada en forma aislada por Trent y Coen (1973), Marsh (1980, 2001), Young (1999), Rinderman y otros (2001). La “interacción” por Feldman (1987), Cajide (1994), García Ramos (1996), Jackson (1999) y Marsh (2001). Como un único factor lo hace Molero y Ruiz (2005), dentro de él evalúan los siguientes aspectos: muestra de interés por el curso, satisfacción por la enseñanza, preocupación por el aprendizaje, disposición y práctica de la objetividad e imparcialidad en el trato con los alumnos entre otros.

\section{Factor 7: Evaluación integral del docente}

En este factor el estudiante evalúa el desempeño general del docente en el curso y en todos los aspectos evaluados anteriormente. Entre los investigadores que identifican esta dimensión se encuentran: Abrami (19990), Tejedor (1990), Mateo y Fernández (1992), Marsh y Roche (1993, 1997) y García Ramos (1996, 1997, 1998).

\section{Tercera Etapa:}

Es el proceso de aplicación del AFC a los modelos de encuesta estudiantil correspondiente a los ciclos académicos del periodo 2008-II (iteración 2) al 2014-I (iteración 13). El objetivo de este proceso fue obtener un modelo cuya validación cumpla con las siguientes características: (a) valor de variancia acumulada mayor al 70\%, (b) que las preguntas diseñadas para medir los factores se correlacionen estadísticamente y tengan interpretación de pertenencia, (c) encuesta estudiantil con 
igual y adecuado número de preguntas por factor y (d) confiabilidad de la encuesta estudiantil con un valor Alfa de Cronbach mayor a 0.90 .

La Tabla 3 corresponde a un modelo de evaluación docente de 7 factores y a una encuesta estudiantil de 25 preguntas. La encuesta fue aplicada a una muestra de 358 estudiantes extraída aleatoriamente de la población de alumnos matriculados en el ciclo académico 2014-I en la UNALM:

Tabla 3. Modelo de encuesta estudiantil con siete factores.

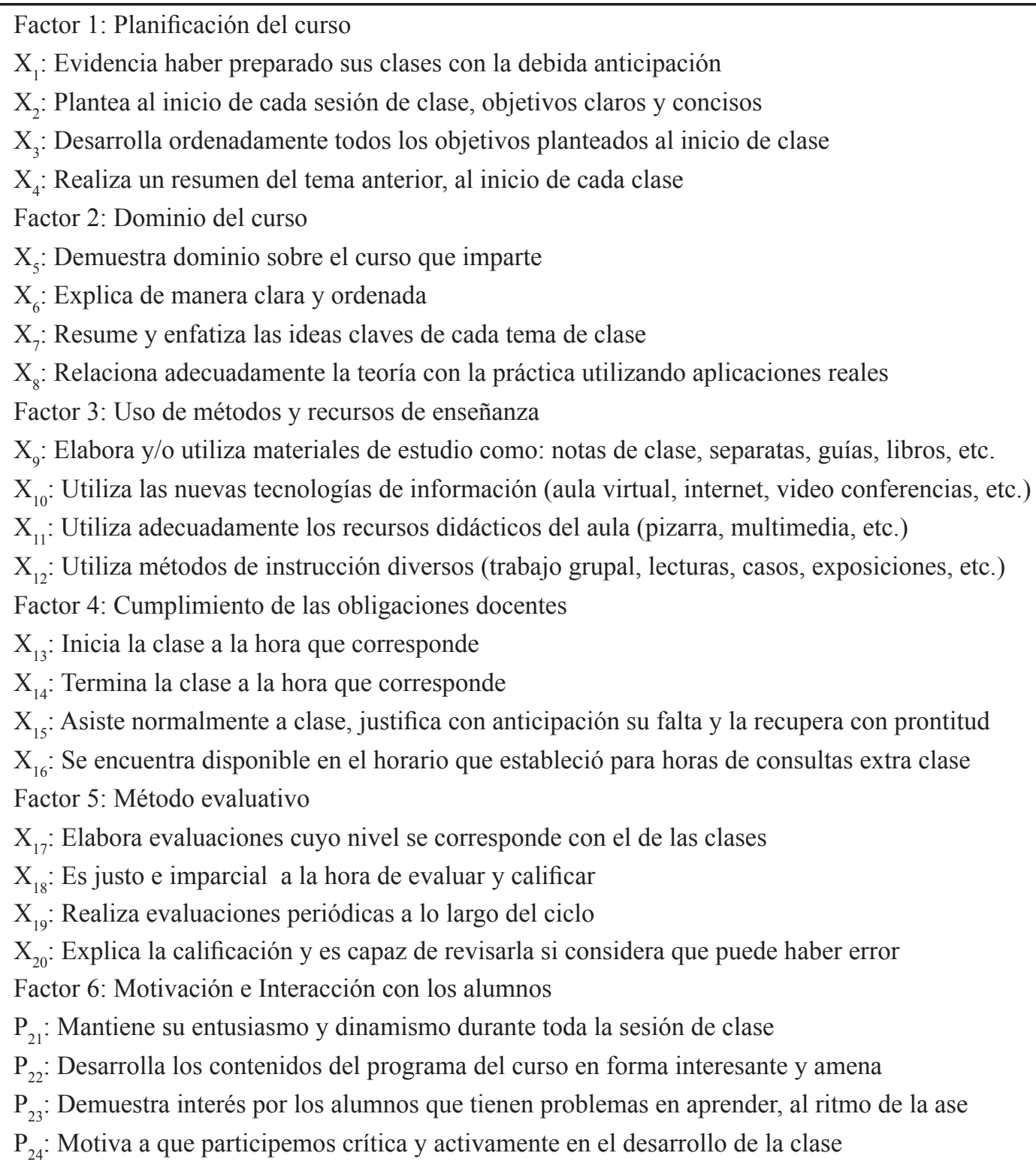

Factor 7: Valoración global

$\underline{\mathrm{P}_{2}}$ : Logró satisfacer mis expectativas y volvería a llevar un curso con él.

Fuente: Elaboración propia

En el proceso de validación del modelo se utilizó AFC. A continuación se detalla los pasos seguidos en la metodología utilizada:

Primer Paso: Objetivo del análisis factorial

Dado que el objetivo del análisis factorial es confirmar que la información subyacente de la evaluación del desempeño docente contenida en la encuesta estudiantil se representa por los siete factores propuestos en el modelo, el objetivo del análisis factorial es principalmente resumir la información.

Segundo paso: Diseño del análisis factorial

Se utiliza la matriz $\mathrm{R}$ porque la agrupación es entre 
variables. Las preguntas son de naturaleza métrica. El número de observaciones por variable es diez veces mayor que el número de variables analizadas.

Tercer Paso: Supuestos del análisis factorial

Secumplenlos supuestosdenormalidad, homocedasticidad y linealidad. El supuesto de multicolinealidad se puede probar a través de diferentes indicadores (Káiser Meyer Oikin, contraste de esfericidad de Bartlett, significancia en la matriz de correlaciones y la medida de suficiencia de muestreo. Para el caso de la presente investigación se utilizaron los tres primeros.

En la Tabla 4 se puede observar que el indicador de Kaiser Meyer Oikin $>0.5$ y que el que el contraste de esfericidad de Bartlett $<0.5$, estos resultados indican el cumplimiento del supuesto de multicolinealidad.

Tabla 4. Test de KMO y Bartlett's.

\begin{tabular}{cc}
\hline Medida/Prueba & Valor \\
\hline $\begin{array}{c}\text { Medida de adecuación maestral de } \\
\text { Kaiser-Meyer-Oikin }\end{array}$ &, 946 \\
Prueba de esfericidad de Barlett &, 000 \\
\hline
\end{tabular}

Fuente: Elaboración propia

El cumplimiento de este supuesto se reafirma con la obtención de un número importante de correlaciones significativas en la matriz de correlaciones.

Cuarto Paso: La estimación de los factores

Se utiliza el método de extracción de componentes principales en la medida que el objetivo principal del AF fue resumir la información original en los siete factores del modelo propuesto. La Tabla 5 se registra para cada uno de los componentes del modelo su autovalor, porcentaje de variancia y porcentaje acumulado de variancia.

Tabla 5. Varianza Total Explicada.

\begin{tabular}{cccc}
\hline Componente & Valores & $\begin{array}{c}\text { Variancia } \\
(\%)\end{array}$ & $\begin{array}{c}\text { Variancia } \\
\text { Acumulada (\%) }\end{array}$ \\
\hline 1 & 11.43 & 45.71 & 45.71 \\
2 & 1.36 & 5.44 & 51.16 \\
3 & 1.25 & 5.00 & 56.14 \\
4 & 1.08 & 4.30 & 60.44 \\
5 & 1.01 & 4.04 & 64.48 \\
6 & 0.89 & 3.56 & 64.05 \\
7 & 0.83 & 3.33 & 71.38 \\
\hline
\end{tabular}

Fuente: Elaboración Propia
En esta tabla se puede observar los cinco primeros componentes tienen autovalores considerados óptimos (mayor a 1) y en conjunto registran un porcentaje de variancia acumulada de $64.44 \%$. Las dos últimas componentes (6 y 7) registran autovalores muy cercanos al óptimo (mayor 0.8) por esta razón se les puede considerar como factores explicativos del modelo y deben mantenerse en el modelo. El porcentaje acumulado de variancia de estos siete factores alcanza un valor de $71 \%$, que se puede considerarse como alto en este tipo de estudios.

Quinto Paso: Interpretación de los factores

Para interpretar los factores y seleccionar la solución factorial definitiva se calculó en primer lugar la matriz inicial de factores no rotados y posteriormente aplicó el método de rotación denominado Normalización Varimax con Kaiser con el fin de lograr soluciones factoriales más simples y teóricamente más significativas.

La matriz de componentes rotados que se muestran en la Tabla 6 permite establecer la asociación entre las variables y los componentes (factores) que participaron en el modelo. Para ello, se aplica el criterio siguiente: se empieza por la primera variable y de izquierda a derecha se identifica a qué factor pertenece la carga factorial más alta, luego se repite el procedimiento hasta llegar al última variable. Este procedimiento estadístico debe ir acompañado de un procedimiento de validación teórico, es decir, la verificación de que la pertenencia estadística expresada por la correlación guarda correspondencia con la pertenencia lógica de la variable en el factor. Esto se comprueba corroborando si la variable fue diseñada inicialmente para medir dicho factor ó en su defecto si existe algún tipo de fundamentación teórica para explicar su asociación con él.

Tabla 6. Matriz de Componentes Rotados.

\begin{tabular}{|c|c|c|c|c|c|c|c|c|}
\hline \multicolumn{9}{|c|}{ Componentes } \\
\hline \multicolumn{3}{|c|}{ Item Variable 1} & 2 & 3 & 4 & 5 & 6 & 7 \\
\hline $\mathrm{P} 1$ & $\mathrm{X}_{23}$ & ,401 & ,217 & ,217 & ,086 & ,119 &, 730 & ,057 \\
\hline $\mathrm{P} 2$ & $\mathrm{X}_{24}$ & ,292 & , 177 &, 120 & ,200 &, 082 & ,773 & ,222 \\
\hline P3 & $\mathrm{X}_{20}$ &, 085 & , 152 & ,244 & ,489 & ,360 &, 425 &, 065 \\
\hline P4 & $X_{4}$ & ,049 &, 017 & ,260 & ,579 & ,206 & ,269 & ,395 \\
\hline P5 & $X_{6}^{4}$ &, 582 & 124 & 434 & 112 , & 299 & 216 & 048 \\
\hline P6 & $X_{10}$ & , 168 & ,220 & ,476 &, 055 &, 036 &,- 036 & ,670 \\
\hline P7 & $X_{1}$ & ,251 & ,213, & 721 , & 242 , & 096, & 190, & 142 \\
\hline P8 & $\mathrm{X}_{7}$ & ,396 & 086 & ,567 &, 322 & ,234 & , 189 & , 102 \\
\hline P9 & $\mathrm{X}_{8}$ & ,464 & , 196 &, 337 & ,394 & ,072 & , 125 & 313 \\
\hline P10 & $\mathrm{X}_{5}$ &, 431 & ,351 & ,496 & ,274 &, 022 & ,252 &, 047 \\
\hline P11 & $\mathrm{X}_{11}$ & ,474 & ,273 & ,526 & ,094 & ,029 & ,016 &, 174 \\
\hline P12 & $X_{12}$ & , 183 & ,118 &,- 035 & 133 & ,348 & ,290 & 678 \\
\hline P13 & $X_{9}$ & ,168 & ,077 & ,407 &, 063 & ,608 &, 165 & ,210 \\
\hline P14 & $\mathrm{X}_{14}$ & , 140 & ,606 & ,420 &,- 070 & ,235 & ,220 &,- 182 \\
\hline P15 & $\mathrm{X}_{16}$ & 100 & 622 & ,205 & ,415 & 071 & 184 & 111 \\
\hline
\end{tabular}


Continuación de tabla 6

\begin{tabular}{lllllllll}
\hline P16 & $X_{2}$ &, 408 &, 214 &, 084 &, 688 &, 138 &, 026 &, 127 \\
P17 & $X_{15}$ &, 330 &, 677 &, 097 &, 188 &, 255 &,- 029 &, 166 \\
P18 & $X_{3}$ &, 574 &, 244 &, 214 &, 397 &, 119 &, 150 &, 026 \\
P19 & $X_{22}$ &, 782 &, 226 &, 244 &, 041 &, 158 &, 261 &, 185 \\
P20 & $X_{13}$ &, 225 &, 739 &, 032 &, 083 &, 071 &, 249 & 220 \\
P21 & $X_{17}$ &, 381 &, 413 &, 044 &, 363 &, 483 &,- 065 &, 107 \\
P22 & $X_{21}$ &, 780 &, 178 &, 123 &, 202 &, 132 &, 223 &, 130 \\
P23 & $X_{25}$ &, 643 &, 233 &, 261 &, 197 & 303 &, 287 &, 050 \\
P24 & $X_{18}$ &, 280 &, 307 &, 200 &, 511 &, 427 &, 168 &,- 138 \\
P25 & $X_{10}$ &, 157 &, 179 &,- 013 &, 199 &, 775 &, 077 &, 154 \\
\hline
\end{tabular}

El Componente 1 contiene con los valores más altos de correlación a la variable $\mathrm{X}_{25}$ (la única diseñada para medir el Factor 7: Apreciación general del docente) y a las variables $\left(\mathrm{X}_{3}, \mathrm{X}_{6}, \mathrm{X}_{8}, \mathrm{X}_{21} \mathrm{y} \mathrm{X}_{22}\right)$ diseñadas para medir los factores restantes, excepto el Factor 6: Método evaluativo. Se recomienda excluir del modelo el Factor 7 por no ser una dimensión diferenciadora en la evaluación docente y mantener la variable $\mathrm{X}_{25}$ como una pregunta de validación final de la encuesta estudiantil.

El Componente 2 contiene con los valor más altos de correlación a las variables $\mathrm{X}_{13}, \mathrm{X}_{14}, \mathrm{X}_{15}, \mathrm{X}_{16}$ diseñadas para medir el Factor 4: Obligaciones docentes. Se recomienda mantener en el modelo estas cuatro preguntas e incluir como pregunta adicional "Devuelve las evaluaciones calificadas en el plazo establecido", la cual se encontró asociada al factor en los modelos precedentes.

El Componente 3 contiene con los valores más altos de correlación a las variables $\mathrm{X}_{5} \mathrm{y}_{\mathrm{X}_{8}}$ diseñadas para medir el Factor 2: Dominio del curso y a la variable $\mathrm{X}_{1}$ diseñada para medir el Factor 1: Planificación del curso. En los modelos precedentes se ha podido observar la existencia de correlación entre estas variables. Se recomienda considerar los Factores 1 y 2 como un solo factor denominado "Planificación y dominio del curso". También, se recomienda mantener en el modelo estas tres variables e incluir las preguntas $\mathrm{X}_{5}$ : Demuestra dominio sobre el curso que imparte y $\mathrm{X}_{6}$ : Explica de manera clara y ordenada, diseñadas para medir estos dos factores y tuvieron el segundo valor más alto de correlación con la Componente 1 la cual se ha sugerido eliminar por estar asociada con éstos dos factores.

Los Componentes 4 y 5 contienen con los valores más altos de correlación a las variables $\mathrm{X}_{17}, \mathrm{X}_{18}, \mathrm{X}_{19} \mathrm{y} \mathrm{X}_{20}$ diseñadas para medir el Factor 5: Método evaluativo. Este resultado indica que en esta última interacción los alumnos de la UNALM perciben diferencias, sin embargo desde el punto de vista teórico y en la práctica con los resultados de las interacciones anteriores éstas preguntas se diseñaron y se encontraron correlacionadas con este factor. Se recomienda considerar dentro del modelo al Factor 5: modelo evaluativo y medirlo por medio de las variables $\mathrm{X}_{17}, \mathrm{X}_{18}, \mathrm{X}_{19} \mathrm{y} \mathrm{X}_{20}$ diseñadas para medir este factor e incluir la pregunta "Realiza evaluaciones periódicas a lo largo del ciclo", asociada a este factor en los modelos precedentes.

El Componente 6 contiene con los valores más altos de correlación a las variables $\mathrm{X}_{23} \mathrm{y} \mathrm{X}_{24}$ diseñadas para medir el Factor 6: Motivación e interacción con los alumnos, y con el segundo valor más alto a las variables $\mathrm{X}_{21} \mathrm{y} \mathrm{X}_{22}$. Se recomienda mantener en el modelo las variables $\mathrm{X}_{21}, \mathrm{X}_{22}$, $\mathrm{X}_{23} \mathrm{y} \mathrm{X}_{24}$ e incluir la pregunta "Propicia una comunicación fluida con los alumnos, creando un clima de confianza", asociada al factor en los modelos precedentes.

El Componente 7 contiene con los valores más altos de correlación a las variables $\mathrm{X}_{10} \mathrm{y} \mathrm{X}_{12}$ diseñadas para medir el Factor 3: Uso de métodos y recursos de enseñanza, con el tercer valor más alto a la variable $\mathrm{X}_{9} \mathrm{y}$ con el cuarto a $\mathrm{X}_{11}$. Se recomienda mantener en el modelo las variables $\mathrm{X}_{10} \mathrm{y}$ $\mathrm{X}_{12}$, modificar las variables $\mathrm{X}_{9} \mathrm{y}_{11}$ a "Elabora y utiliza materiales de estudio diversos: notas de clase, separatas, libros, etc." y "Utiliza recursos de enseñanza diversos: pizarra, multimedia, libros, etc." Respectivamente, e incluir la pregunta "Realiza un resumen del tema anterior, al inicio de cada clase"

En la Tabla 7 se muestra el modelo sugerido para adoptar en la UNALM constituido por 5 factores: (1) Planificación y dominio del curso, (2) Métodos y recursos de enseñanza, (3) Obligaciones docentes, (4) Método evaluativo, (5) Motivación e interacción con los alumnos. También se muestra la propuesta encuesta estudiantil (26 preguntas) que se puede utilizar como instrumento de medición.

Tabla 7. Modelo de Encuesta Estudiantil.

Factor 1: Planificación y dominio del curso

$\mathrm{P}_{1}$ : Evidencia haber preparado sus clases con la debida anticipación

$\mathrm{P}_{2}$ : Relaciona adecuadamente la teoría con la práctica utilizando casos reales

$\mathrm{P}_{3}$ : Demuestra dominio sobre el curso que imparte

$\mathrm{P}_{4}$ : Explica de manera clara y ordenada

$\mathrm{P}_{5}$ : Resume y enfatiza las ideas claves de cada tema de clase

Factor 2: Métodos y recursos de enseñanza

$\mathrm{P}_{6}$ : Realiza un resumen del tema anterior, al inicio de cada clase

$\mathrm{P}_{7}$ : Elabora y utiliza materiales de estudio diversos como: notas de clase, separatas, libros, etc.

$\mathrm{P}_{8}$ : Utiliza las nuevas tecnologías de información: aula virtual, internet, video conferencias, etc.

$\mathrm{P}_{9}$ : Utiliza recursos de enseñanza diversos: pizarra, multimedia, libros, etc.

$\mathrm{P}_{10}$ : Utiliza métodos de enseñanza diversos: trabajo grupal, problemas, casos, etc. 
Continuación de tabla 7

Factor 3: Obligaciones docentes

$\mathrm{P}_{11}$ : Inicia la clase a la hora que corresponde

$\mathrm{P}_{12}$ : Termina la clase a la hora que corresponde

$\mathrm{P}_{13}$ : Asiste normalmente a clase, justifica con anticipación su falta y la recupera con prontitud

$\mathrm{P}_{14}$ : Se encuentra disponible en el horario que estableció para horas de consultas extra clase

$\mathrm{P}_{15}$ : Devuelve las evaluaciones calificadas en el plazo establecido

Factor 4: Método evaluativo

$\mathrm{P}_{16}$ : Elabora evaluaciones cuyo nivel se corresponde con el de las clases

$\mathrm{P}_{17}$ : Es justo e imparcial a la hora de evaluar y calificar

$\mathrm{P}_{18}$ : Realiza evaluaciones periódicas a lo largo del ciclo

$\mathrm{P}_{19}$ : Explica la calificación y es capaz de revisarla si considera que puede haber error

$\mathrm{P}_{20}$ : Aplica evaluaciones que condujeron a mejorar mi aprendizaje

Factor 5: Motivación e interacción con los alumnos

$\mathrm{P}_{21}$ : Propicia una comunicación fluida con los alumnos, creando un clima de confianza

$\mathrm{P}_{22}$ : Mantiene su entusiasmo y dinamismo durante toda la sesión de clase

$\mathrm{P}_{23}$ : Demuestra interés por los alumnos que tienen problemas en aprender, al ritmo de la clase

$\mathrm{P}_{24}$ : Motiva a que participemos crítica y activamente en el desarrollo de la clase

$\mathrm{P}_{25}$ : Desarrolla los contenidos del programa del curso en forma interesante y amena

Valoración global

$\mathrm{P}_{26}$ : Logró satisfacer mis expectativas y volvería a llevar un curso con él

Fuente: Elaboración propia

\section{Consideraciones finales:}

A la luz de la revisión bibliográfica realizada durante la investigación se puede llegar a las siguientes consideraciones finales:

La evaluación del desempeño docente en el ámbito de la enseñanza aprendizaje es un procedimiento que emplean las universidades para fomentar mejoras en la actividad docente y por ende repercuten en la calidad educativa de la institución a la cual pertenece.

En la actualidad no existe un modelo único de evaluación del desempeño docente universitario, difieren en el número y operacionalización de los factores que intervienen, por la fuente utilizada para realizar la evaluación y en la confiabilidad y validez de los instrumentos que permiten efectuar la medición.

La evaluación del desempeño docente debe ser parte de un modelo integral de evaluación que contemple no sólo la evaluación en el ámbito instructivo, sino también a todas las otras actividades que desarrollan los docentes a lo largo de su trayectoria académica.

\section{Conclusiones}

El modelo de siete factores: (1) Planificación del curso, (2) dominio del curso, (3) Uso de métodos y recursos de instrucción, (4) Cumplimiento de obligaciones docentes, (5) Método evaluativo, (6) Motivación e interacción con los alumnos y (7) Valoración global, presenta mayor validez que el modelo utilizado actualmente en la UNALM constituido por cinco factores:(1) Conducción del aprendizaje, (2) Disposición y cumplimiento, (3) Motivación, (4) Evaluación y (5) Uso de material educativo.

El modelo alcanza un porcentaje de variancia acumulado de $71.36 \%$. La confiabilidad de la encuesta estudiantil registra un Coeficiente Alfa de Cronbach de 0.9336.

La dimensión del modelo denominado "Valoración global (Factor 7) no es un factor diferenciador dentro del modelo por lo que puede ser eliminado y considerar la variable que la mide como una pregunta de apreciación general.

Las dimensiones del modelo identificados como "Planificación del curso Factor (Factor 1)" y "Dominio del curso (Factor 2)" se encuentran estrechamente correlacionados por lo que se les puede considerar como un único factor el cual pude ser etiquetado como "Planificación y dominio del curso".

\section{Recomendaciones}

Se recomienda validar y adoptar un modelo de cinco factores integrado por las siguientes dimensiones de evaluación docente: (1) Planificación y dominio del curso, (2) Métodos y recursos de instrucción, (3) Obligaciones docentes, (4) Método evaluativo, (5) Motivación e interacción con los alumnos.

Se recomienda utilizar una encuesta estudiantil aplicada en forma presencial y constituida por 26 preguntas, donde cada factor sea medido por 5 preguntas y 1 pregunta sea utilizada a modo de valoración global de la apreciación estudiantil del desempeño docente.

Se recomienda validar la encuesta estudiantil cuando se aplica a través de internet.

Se recomienda desarrollar nuevos instrumentos que permitan evaluar la enseñanza desde ámbitos diferentes al instructivo, así como para evaluar las otras actividades de la actividad docente. 


\section{Literatura citada}

Acevedo, R. (2003). "Factores que inciden en la Competencia Docente Universitaria: Un Modelo Jerárquico Lineal". Tesis de grado de doctor en la Universidad Complutense de Madrid. España

Ansión, J., Guzmán L. y Korswagen, R. (1998). "Gestión Docente Universitaria Modelos Comparados". Volumen 2. Chile, pp. 129-161.

De Mata, D. (1998). "Gestión Docente Universitaria Modelos Comparados". Volumen 2. Chile, pp. 163-174.

Fresa, M. y Vera, Y. (1998). “Evaluación del Desempeño del Personal Académico: Análisis y Propuesta de Metodología Básica”. ANUIES. México, pp. 1-207.

Hair, J., Anderson, R., Tatham, R. and Black, W. (1999). "Análisis Multivariante". 5ta edición. Madrid, pp. $79-140$

Molero D. y Ruiz J. (2005). "La Evaluación de la Docencia Universitaria. Dimensiones y Variables más Relevantes". Revista de Investigación Electrónica, Volumen 23, Número 1, pp. 57-84

Muñoz J.M., Ríos M.P. y Abalde E. (2002). "Evaluación Docente vs. Educación de la Calidad". Revista Electrónica de Investigación y Evaluación Educativa, Volumen 8, Número 2. http://w.w.w.uv.es/RELIEVE/ v8n2/RELIEVEv8n2_4.htm

Rosas, F. y Moreno, A. (1998). "Las Técnicas Multivariantes Aplicadas a la Identificación de Atributos Asociados a Universidades". Tesis para la obtención del título de Ingeniero Estadístico, Universidad Nacional Agraria La Molina. Lima.

Valdés Veloz, H. (2000). "Evaluación del Desempeño Docente". Ponencia en el Encuentro Iberoamericano sobre Evaluación del Desempeño Docente. Ciudad de México, 23-25 de mayo, pp. 1-20.

Villarreal Gutiérrez, M. (1990). "Evaluación Universitaria: Evaluación de Catedráticos". México, pp. 77-103. 